\title{
Orthopaedic anatomy of the sternum: A micro-CT study to guide the fixation of sternal fractures
}

\author{
Jonathon Lindner ${ }^{*}$, Lonnie Douglas ${ }^{1}$, David Seligson ${ }^{1}$, Seid Waddell ${ }^{1}$ and Michael Voor ${ }^{2}$ \\ ${ }^{1}$ University of Louisville Department of Orthopaedic Surgery, USA \\ ${ }^{2}$ University of Louisville Departments of Orthopaedic Surgery and Bioengineering, USA
}

\begin{abstract}
Background: The rise of manubriosternal joint (MSJ) fractures and lack of literature has spurred an interest in surgical fixation. We hypothesize that locations exist within the sternum that will yield best screw purchase and more rigid fixation of sternal fractures.

Methods: Nine cadaveric sternums were scanned using micro-CT and reconstructed. The anterior surface length and angle were measured. The sternum was divided into percentages from the jugular notch (0\%) to the xiphisternal joint (100\%). Three reference points were marked dividing the axial section into 4 equal quarters from right to left and the total width was measured. At each point of interest, total and anterior and posterior cortical thickness were measured. Values at each percentage were averaged and analyzed.

Results: The average length of the manubrium and sternum were $5.1 \mathrm{~cm}$ and $9.8 \mathrm{~cm}$ respectively and the MSJ occurred near $35 \%$. The sternal angle averaged $16.9^{\circ}$. The sternum was widest at $10 \%$, averaging $6.6 \mathrm{~cm}$ and narrowest at the MSJ. The superolateral and inferomedial manubrium and superomedial sternum was the thickest. Additionally, the middle $50 \%$ of the manubrium and the superior sternal body exhibited small specimen variations and had small standard errors. The $95 \%$ confidence interval encompasses a maximum and minimum thickness value of 21.4 and $5.2 \mathrm{~mm}$ respectively. The results obtained, though clinically relevant, exhibited no statistical difference.

Conclusions: Micro-CT is an effective tool for the evaluation of sternal anatomy. The middle $50 \%$ of the manubrium and superior sternal body exhibited the least variability and should be utilized for screw placement during fracture fixation. Screw lengths should be no greater than 21 mm and no less than 5.2 mm. This study predicts that the sternum is amenable to effective plate and screw fixation. A larger scale CT study of living patients warranted before areas of greatest screw purchase can be determined with certainty.
\end{abstract}

\section{Background}

The Angle of Louis, more commonly known as the sternal angle or the manubriosternal joint (MSJ), was first described by Pierre Charles Alexandre Louis, a $19^{\text {th }}$ century Frenchman who postulated that increased angulation was associated with worsening progression of emphysema [1]. Since then, the anatomy of the sternum and the MSJ has remained essentially unstudied. This can be attributed to a multitude of factors, the most important of which is the historical infrequency of sternal fractures and low complication rates of cerclage wiring following median sternotomy for open thoracic surgery [2]. Today, sternal fractures are believed to be present in $0.64 \%$ of all motor vehicle trauma patients [3]. Furthermore, sternal fractures are present in $3 \%$ to $8 \%$ of all blunt force trauma patients [2].

The sternum is comprised of three bones: the manubrium, the sternal body, and the xiphoid process. It articulates with the clavicles and the cartilaginous portions of the first through seventh ribs and serves as a major attachment for the sternocleidomastoid and pectoralis major muscles. The MSJ, found at the insertion of the second rib, is connected by fibrocartilage [4]. It is comprised of cancellous bone encased in a thin layer of compact cortical bone. The anterior surface of the manubrium and proximal sternal body is convex and the posterior surface is concave. Further down the body, the shape transitions to biconcave before reaching the xiphoid [5]. These known anatomical properties of the sternum help explain an inherent weakness at the
MSJ. In fact, Labbe et al (2009) found that $82 \%$ of sternal fractures were located at the MSJ and of those $56 \%$ were fracture-dislocations [6].

Recently, there has been increasing interest in fixation of MSJ fractures and dislocations due to increasing incidence of the fractures themselves combined with the desire to avoid the comorbidities of poorly healed fractures. This incidence has spiked in the last few decades as a result of increasing emphasis on seat belt usage and growing participation in contact sports. Also, the widespread use of CT imaging of trauma patients in emergency departments has increased the apparent incidence [3,4,7]. The indications for operative management include nonunion, chronic pain leading to respiratory compromise, damage to underlying organs, and failed closed reduction of displaced fractures [8]. However, at this time there has been no standard method proven to be superior to any other for the fixation of MSJ fracture-dislocations $[9,10]$. All comparative studies to date have focused primarily on sternal reduction following median sternotomy. In these studies, plating has been proven to provide lower rates of nonunion and mediastinitis, reduced pain, increased safety, closer sternal approximation, and required minimal soft tissue dissection

Correspondence to: Jonathon R. Lindner M.D, M.S.E., 550 S. Jackson St., 1st Floor ACB, Louisville, KY 40202, USA; Tel: (502) 553-4963; E-mail: jrlin718@ gmail.com

Received: April 14, 2017; Accepted: April 20, 2017; Published: April 29, 2017 
as compared to cerclage wiring $[2,11,12]$. Additionally, preliminary studies of plate fixation of MSJ fracture-dislocations have shown low rates of complications and infection, excellent healing, and minimal damage to surrounding structures and vascular supply $[10,13,14]$. These studies along with the overwhelming success of rigid plate fixation in other orthopedic applications, place this method in the forefront for fixation of displaced MSJ fractures.

The rising incidence of MSJ fracture-dislocations, lack of standardized fixation method, relative lack of anatomical knowledge, and the promising results of plate fixation warrant an anatomical study of the sternum. The objective of this study was to determine the general anatomy, areas of greatest total and cortical thickness, and regions with the least variation of cadaveric sternums using micro-CT imaging. Thickness patterns were used to determine if the sternum is amenable to plate fixation and will serve to guide the placement of screws.

\section{Materials and methods}

Cadaveric specimens with attached clavicles and ribs were obtained from the University of Louisville Fresh Tissue Dissection Laboratory. Each specimen was lightly embalmed using the technique described by Anderson [15]. Once obtained, the specimens were sealed and frozen at $-20^{\circ} \mathrm{C}$ until scanned in groups of three in a cylinder with diameter of $175 \mathrm{~mm}$.

\section{Exclusion criteria:}

1 Traumatic cause of death

\section{History of open thoracic surgery}

\section{Previous sternal fracture}

\section{CPR performed prior to death}

An ACTIS 150/225 FFi-HR CT/DR System, with the FeinFocus $225 \mathrm{kV}$ x-ray source (Bio-Imaging Research Inc., Lincolnshire, IL) was used for all micro-CT sternal scans. The $\mathrm{x}$-ray source and image intensifier were fixed $703 \mathrm{~mm}$ apart in this cone beam scanner. The rotational and vertical displacement mechanisms were driven by Actis Software to manipulate the samples during scanning. The gantry was set with the source-image distance and offset distance to obtain the desired final image magnification. Each scan was performed at $55 \mathrm{kV}$ and $146 \mu \mathrm{A}$ power, with 1000 views/rotation. Each view was captured twice and averaged for noise suppression. The resulting scans produced a nominal voxel-size resolution of $85 \mu \mathrm{m}$. The complete image stack was imported into a 3-D voxel-based modeling software (Volume Graphics VGStudio Max 2.2, Heidelberg, Germany) to create a solid model where density (gray-scale) thresholds were set to remove the soft tissues from the images, leaving only the bone tissue (Figure 1A).

Within the 3-D software, the sternal length was determined using a 3-point measuring system on the midsagittal slice from the jugular notch of the manubrium to the sternal angle and then to the xiphisternal joint (XSJ) along the anterior surface of the sternum (Figure $2 \mathrm{~A}$ ). The xiphoid process was excluded in all analyses because of its high degree of anatomical and age related variability and mobility. The sternal angle was determined using a 4-point angle tool with one line resting tangential to the manubrium and the other tangential to the body of the sternum (Figure 2B).

Using the sternal length measurement previously acquired, the sternum was divided into percentages. Measurements were taken every $10 \%$ in a true axial plane from the jugular notch (0\%) to the XSJ (100\%) along the length of the sternum. At each percentage in the axial plane,

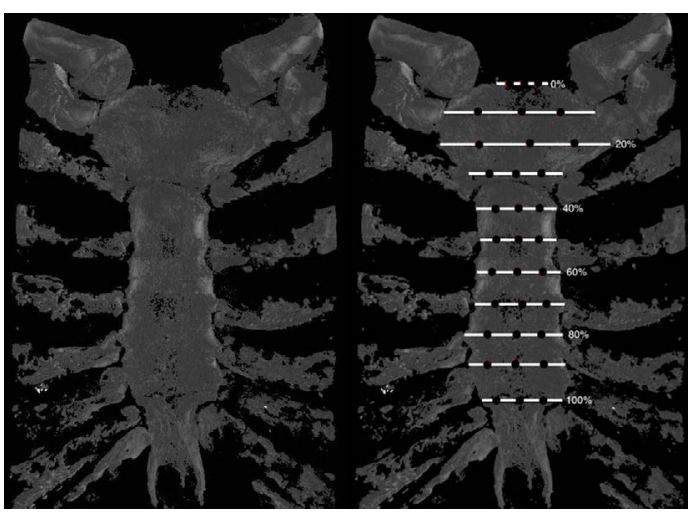

Figure 1. A. 3-D reconstruction of a sternum micro-CT scan with density thresholds set to remove soft tissues. The scan includes the entire sternum, attached clavicles, and associated ribs. B. The white lines indicate the areas of interest measured every $10 \%$ from the jugular notch $(0 \%)$ to the xiphisternal joint $(100 \%)$. The dots on each line indicate areas where total and cortical thickness measurements were made dividing each axial slice into four equal quarters.

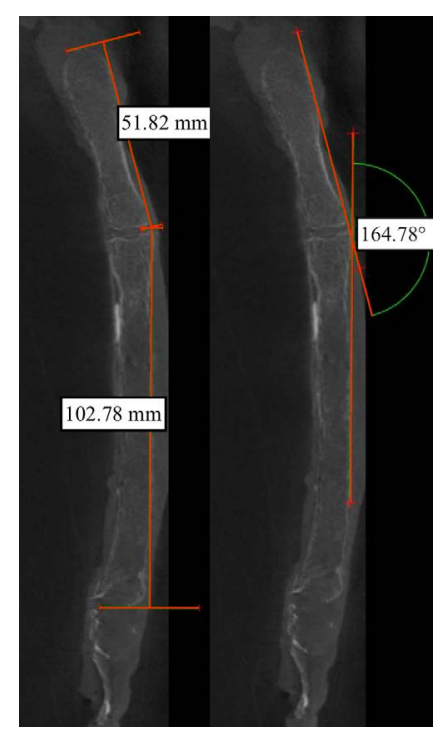

Figure 2. Midsagittal view of a sternal micro-CT scan. A. Two calipers were used to measure the anterior surface length of the manubrium and the sternal body. Measurements were from the jugular notch to the manubriosternal joint and then to the xiphisternal joint. B. A four-point angle tool was used to measure the manubriosternal angle. One line lies tangential to the anterior manubrial surface and the other lies tangential to the anterior sternal body surface.

three reference points were marked dividing the axial section into 4 equal quarters from right to left (Figure 1B). At each of these locations, width, total thickness, and anterior and posterior cortical thickness were measured (Figure 3 ). In the event of a small discontinuity in cortical bone present at a given location, measurements were made using a linear approximation from bone immediately adjacent to the reference point. In the event of large discontinuities in cortical bone, the cortical thickness at that point was recorded to be $0 \mathrm{~mm}$.

\section{Results}

A total of 9 sternums were scanned and analyzed: 7 males and 2 females. Due to the use of cadaveric specimens, there was an uneven age distribution with an average age of 75.1 years and a range of 48 to 92 years. Measurement results are given as mean (S.D.). The average anterior surface length of the manubrium and the sternal body were $5.1 \mathrm{~cm} \mathrm{(0.8)} \mathrm{and} 9.8 \mathrm{~cm}(1.3)$ respectively. The manubrium was almost always contained within the first $35 \%$ of the specimen. That led to 


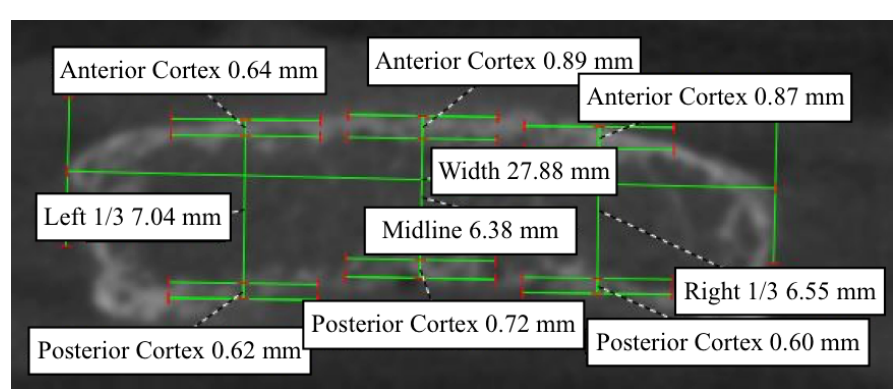

Figure 3. Axial slice of a micro-CT scan of the manubrium. At each point of interest, tota width, right third, left third, and midline total thickness, and anterior and posterior cortical thickness were measured.

decreased reliability and large variability of the resulting measurements at the 30 and $40 \%$ marks. The average sternal angle was $16.9^{\circ}(7.4)$ and exhibited a high degree of variability among specimens.

The manubrium was widest at the $10 \%$ mark, averaging $6.6 \mathrm{~cm}$ (0.9). The sternum was narrowest at the MSJ and at the XSJ, which by the numerical system employed was always at the $100 \%$ mark (Figure 4). The total sternal thickness was greater at the right and left thirds than the midline for the first $10 \%$. From $20 \%$ to $40 \%$ the overall thickness was greater at midline. The midline and peripheral thicknesses were almost equal at the $50 \%$ mark and beyond that the peripheral thicknesses were greater (Figure 5A). The cortical thicknesses were not as clearly defined, as there was greater variation between the right and left third thicknesses. However, the midline cortical thickness was greater from $10 \%$ to $40 \%$ and at $100 \%$ and the peripheral cortical thicknesses were greater at $0 \%, 50 \%$, and from $70 \%$ to $90 \%$. At $60 \%$ the midline cortex thickness was between the left and right third thicknesses with the left being thicker than midline and the right being thinner than midline (Figure 5B).

The sternum was thickest at the periphery at the $10 \%$ mark with an average thickness of $1.5 \mathrm{~cm} \mathrm{(0.3)} \mathrm{and} \mathrm{the} 95 \%$ confidence interval giving a maximum value of $21.1 \mathrm{~cm}$. It was thinnest at the $20 \%$ mark peripherally with a thickness of $8.5 \mathrm{~mm}$ (1.7) with a 95\% confidence interval giving a minimum value of $5.2 \mathrm{~mm}$. Though interesting, and possibly useful clinically, as will be discussed, these patterns were not found to be statistically significant.

\section{Discussion}

The incidence of sternal fractures is low. Sternal fractures necessitating surgical fixation are even more infrequent. However, when an orthopedic surgeon is required to address sternal fractures operatively, the paucity of literature guidance for both preoperative planning and intraoperative technique is suboptimal. In the current setting the surgeon is often forced to improvise.

This cadaveric study describes the anatomy of the manubrium and sternum. The use of micro-CT has been shown to be a good method of evaluating cortical bone mass and predicting areas of likely propagation of fractures of other bones [16,17]. Micro-CT of the sternum produced high-resolution images and 3-D models allowing for accurate determination of the bony surface as well as cortical mass and anatomic variations.

Previous studies have shown significant size differences between male and female clavicles [18]. This characteristic of female thoracic anatomy predicts smaller female sternums. Female sternums in this study were shorter, thinner, narrower, and had a lower percentage of cortical bone compared to their male counterparts. This is likely attributable to both morphological and age-related sex differences.

Rigid plate fixation has been shown to be an effective method of fixation of MSJ and other sternal fractures [2,7,10,11,13,14,19]. Ostensibly, the close proximity of vital organs directly abutting the dorsal surface of the sternum makes substantive bicortical constructs unfeasible. Additionally, both cortical and overall bone thickness is relatively thin. Due to these conditions very short screws must be used. Obtaining strong, safe fixation is challenging. These problems can be overcome to some extent by the use of screws with decreased pitch and increased thread depth [20,21].

Despite the age related changes and cortical thinning seen in these cadaveric specimens, this study shows that the manubrium and sternum still do lend themselves to fixation by plate and screws. The majority of the patients with these fractures are contact sports athletes and those involved in motor vehicle collisions and consequently are younger than the cadaveric specimens examined [3,4,7]. Younger patients have greater cortical and trabecular bone mass making them even better candidates for screw and plate fixation. Assuming the bone patterns identified in these elderly patients are consistent in a younger population, we can begin to predict the areas that will allow the greatest screw purchase and subsequent greatest fixation. Based on this assumption, the superolateral and inferomedial manubrium and superomedial and inferolateral sternal body would provide the greatest purchase. Additionally, the middle portion of the manubrium, as well as the proximal sternal body exhibit little specimen variation and have small standard errors. Consequently, those areas should be utilized for screw placement during fracture fixation.

It is safe to say that the maximum screw length into bone required should be no longer than $21.4 \mathrm{~mm}$. This does not include the length of the screw head or shaft that lies embedded within the plate. Screws longer than $21.4 \mathrm{~mm}$ would likely pose a threat to vital organs beneath the sternum. Currently there is no literature suggesting a maximum safe value for posterior screw protrusion. Screws shorter than $5.2 \mathrm{~mm}$ can be assumed to be safe in all areas of the manubrium and sternum but would likely be unicortical. The results at $0,30,40$ and $100 \%$ showed the greatest variation in all measurements. This is inherent to the measuring system employed and the fact that the MSJ, known for

\section{Width}

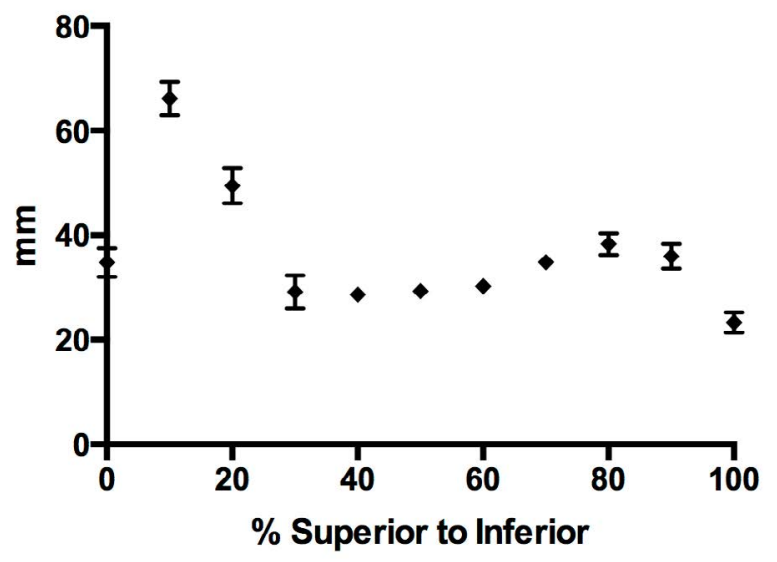

Figure 4. Maximum sternal width in $\mathrm{mm}$ along the length of the sternum from the jugular notch $(0 \%)$ to the xiphisternal joint $(100 \%)$. Error bars represent the standard error of the mean. 


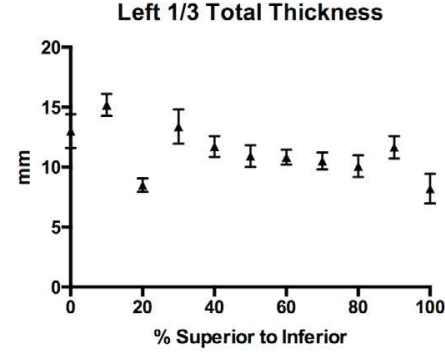

Left $1 / 3$ Cortical Thickness

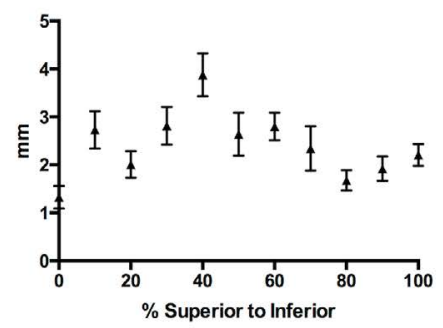

Midline Total Thickness

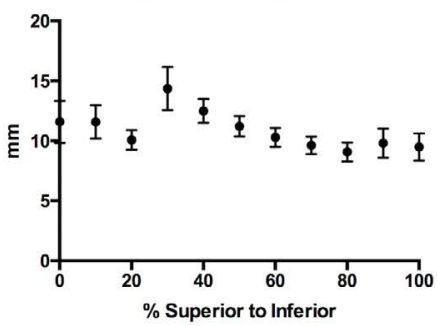

Midline Cortical Thickness

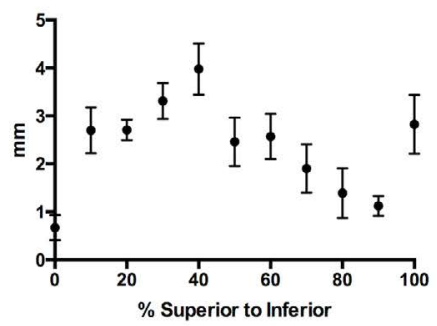

Right 1/3 Total Thickness

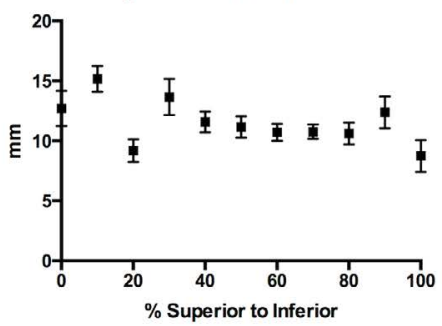

Right 1/3 Cortical Thickness

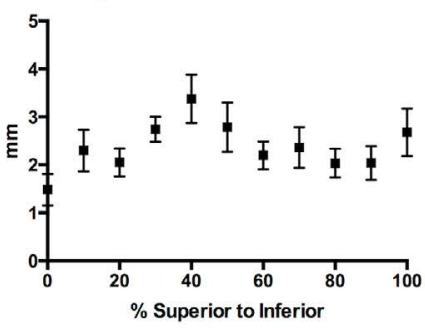

Figure 5. A Total sternal thickness and B. total sternal cortical thickness in $\mathrm{mm}$ at the left and right thirds and midline along the length of the sternum from the jugular notch ( $0 \%)$ to the xiphisternal joint $(100 \%)$. Error bars represent the standard error of the mean.

its wide anatomical and age related variability, always fell between 30 and $40 \%$. These areas should be avoided when placing screws.

This study further defines the bony anatomy of the sternum. Unfortunately, the use of micro-CT is limited at this time to small cadaveric specimens and consequently results in older specimen age and less sternum availability. A larger scale CT study of living patients with a normal age and equal gender distribution is warranted before areas of greatest screw purchase can be determined with certainty. In addition, the success of preliminary trials of rigid plate fixation for displaced MSJ and other sternal fractures show promise for development of an orthopedic standard for sternal fracture fixation $[2,7,10,11,13,14,19]$.

\section{Acknowledgements}

This study was made possible by funding from the Fischer-Owen Orthopaedic Trust Fund from The University of Louisville Department of Orthopaedic Surgery.

\section{References}

1. Coscione A, Dixon L, Ellis H. The “Angle of Louis”. Eur J Anat 17: 190-192.

2. Nazerali RS, Hinchcliff K, Wong MS (2014) Rigid fixation for the prevention and treatment of sternal complications. Ann Plast Surg 72 Suppl 1: S27-30. [Crossref]

3. Knobloch K, Wagner S, Haasper C, Probst C, Krettek C et al. (2006) Sternal fractures occur most often in old cars to seat-belted drivers without any airbag often with concomitant spinal injuries: clinical findings and technical collision variables among 42,055 crash victims. Ann Thorac Surg 82: 444-450. [Crossref]

4. Hayashi D, Roemer FW, Kohler R, Guermazi A, Gebers C et al. (2014) Thoracic injuries in professional rugby players: mechanisms of injury and imaging characteristics. $\mathrm{BrJ}$ Sports Med 48: 1097-1101. [Crossref]

5. Drake R, Vogl AW, Mitchell A. Gray's Anatomy for Students. Philadelphia, PA: Elsevier; 2010.5.

6. Labbe JL, Peres O, Leclair O, Goulon R, Scemama P et al. (2009) Fractures of the upper transthoracic cage. J Bone Joint Surg Br 91: 91-96. [Crossref]

7. Richardson JD, Franklin GA, Heffley S, Seligson D (2007) Operative fixation of chest wall fractures: an underused procedure? Am Surg 73: 591-596. [Crossref]

8. Sarkar PK, Counsell D, Rabbi F (1993) Fractures of the sternal body. Ann R Coll Surg Engl 75: 213-214. [Crossref]
9. Harston A, Roberts C (2011) Fixation of sternal fractures: a systematic review. $J$ Trauma 71: 1875-1879. [Crossref]

10. Ergene G, Tulay CM, Anas $\ddot{A} \pm \mathrm{z}$ H (2013) Sternal fixation with nonspecific plate. Ann Thorac Cardiovasc Surg 19: 364-367. [Crossref]

11. Hendrickson SC, Koger KE, Morea CJ, Aponte RL, Smith PK, et al. (1996) Sternal plating for the treatment of sternal nonunion. Ann Thorac Surg 62: 512-518. [Crossref]

12. Wu LC, Renucci JD, Song DH (2005) Sternal nonunion: a review of current treatments and a new method of rigid fixation. Ann Plast Surg 54: 55-58. [Crossref]

13. Gloyer MA, Frei HC, Hotz TK, Kach KP (2011) Osteosynthesis of traumatic manubriosternal dislocations and sternal fractures with a $3.5 / 4.0 \mathrm{~mm}$ fixed-angle plate (LCP). Arch Orthop Trauma Surg 131: 1261-1266. [Crossref]

14. Bonney S, Lenczner E, Harvey EJ (2004) Sternal Fractures: Anterior Plating Rationale. The Journal of Trauma: Injury, Infection, and Critical Care 57: 1344-1346.

15. Anderson SD (2006) Practical light embalming technique for use in the surgical fresh tissue dissection laboratory. Clin Anat 19: 8-11. [Crossref]

16. Senck S, Plank B, Kastner J, Ramadani F, Trieb K et al. (2015) [Visualization of local cortical defects in Charcot foot using microcomputed tomography.]. Orthopade 44: $8-13$ [Crossref]

17. Bala Y, Bui QM, Wang X, Iuliano S, Wang Q et al. (2014) Trabecular and Cortical Microstructure and Fragility of the Distal Radius in Women. Journal of bone and mineral research : the official journal of the American Society for Bone and Mineral Research. 2014

18. Bernat A, Huysmans T, Van Glabbeek F, Sijbers J, Gielen J et al. (2014) The anatomy of the clavicle: A three-dimensional cadaveric study. Clinical anatomy 27:712-723. [Crossref]

19. Divisi D, Di Leonardo G, Crisci R (2013) Surgical management of traumatic isolated sternal fracture and manubriosternal dislocation. J Trauma Acute Care Surg 75: 824829. [Crossref]

20. Chapman JR, Harrington RM, Lee KM, Anderson PA, Tencer AF et al. (1996) Factors affecting the pullout strength of cancellous bone screws. J Biomech Eng 118: 391-398. [Crossref]

21. Bertin KC, Rice RS, Doty DB, Jones KW (2002) Repair of transverse sternal nonunions using metal plates and autogenous bone graft. Ann Thorac Surg 73: 1661-1662. [Crossref]

Copyright: (C)2017 Lindner J. This is an open-access article distributed under the terms of the Creative Commons Attribution License, which permits unrestricted use, distribution, and reproduction in any medium, provided the original author and source are credited. 